A molecular dynamics study of the bee venom melittin in aqueous solution, in methanol, and inserted in a phospholipid bilayer

\author{
Journal Article \\ Author(s): \\ Glättli, Alice; Chandrasekhar, Indira; van Gunsteren, Wilfred F. \\ Publication date: \\ 2006-02 \\ Permanent link: \\ https://doi.org/10.3929/ethz-b-000000388 \\ Rights / license: \\ In Copyright - Non-Commercial Use Permitted \\ Originally published in: \\ European Biophysics Journal 35(3), https://doi.org/10.1007/s00249-005-0033-7
}


Alice Glättli • Indira Chandrasekhar

Wilfred F. van. Gunsteren

\section{A molecular dynamics study of the bee venom melittin in aqueous solution, in methanol, and inserted in a phospholipid bilayer}

Received: 27 June 2005/ Revised: 5 October 2005 / Accepted: 8 October 2005/ Published online: 2 December 2005

(C) EBSA 2005

\begin{abstract}
The structural properties of melittin, a small amphipathic peptide found in the bee venom, are investigated in three different environments by molecular dynamics simulation. Long simulations have been performed for monomeric melittin solvated in water, in methanol, and shorter ones for melittin inserted in a dimyristoylphosphatidylcholine bilayer. The resulting trajectories were analysed in terms of structural properties of the peptide and compared to the available NMR data. While in water and methanol solution melittin is observed to partly unfold, the peptide retains its structure when embedded in a lipid bilayer. The latter simulation shows good agreement with the experimentally derived ${ }^{3} \mathrm{~J}$-coupling constants. Generally, it appears that higher the stability of the helical conformation of melittin, lower is the dielectric permittivity of the environment. In addition, peptide-lipid interactions were investigated showing that the C-terminus of the peptide provides an anchor to the lipid bilayer by forming hydrogen bonds with the lipid head groups.
\end{abstract}

\section{Introduction}

Small, mostly amphiphilic, $\alpha$-helical peptides accomplish simple antimicrobial defence tasks in a number of organisms [1]. Most of these host-defence peptides, such as toxins and antimicrobial peptides, exert their action through permeabilisation of cellular membranes by association with the lipid headgroups [2-5]. They initially adsorb onto the membrane surface adopting a parallel orientation to the bilayer surface and cause an

A. Glättli · I. Chandrasekhar · W. F. van. Gunsteren $(\bowtie)$

Laboratorium für Physikalische Chemie,

Swiss Federal Institute of Technology,

ETH Hönggerberg HCI,

8093 Zürich, Switzerland

E-mail: wfvgn@igc.phys.chem.ethz.ch

Fax: + 41-1-6321039 increase in membrane permeability through disruption of the packing of lipid molecules, which is called the "carpet effect" $[6,7]$. However, they may also reorient to adopt an orientation perpendicular to the bilayer surface, a so-called transmembrane orientation, and then associate to form either barrel-stave pores [8] or toroidal pores $[9,10]$ within the bilayer causing size-selective leakage and eventually membrane-lysis [4, 11]. The mode and effectiveness of the disruption of the membrane barrier function depends on the one hand on the peptide concentration and on the other hand on the membrane lipid composition, but also on factors like hydration level and $\mathrm{pH}[2,12]$. The characteristics and biological activity of some of these peptides is not only of great interest per se, but they also provide model systems for large membrane proteins. As the majority of membrane proteins appears to be composed of amphiphilic transmembrane helices, the investigation of membrane-active peptides that form single membranespanning helices contributes to the understanding of the lipid-protein interactions and the factors that stabilise the structure of membrane proteins.

Melittin, is one of the best studied membrane-interactive polypeptides. It is the major component in the venom of the European honey bee Apis mellifera, has a powerful hemolytic activity [13] and is reported to activate phospholipase $A_{2}$ found in the bee venom $[14,15]$. Furthermore, melittin can induce bilayer fusion [16]. The cationic amphiphilic polypeptide consists of 26 amino acids with the sequence [17]

$$
\begin{aligned}
& \text { Gly }^{1}-\mathrm{Ile}^{2}-\mathrm{Gly}^{3}-\mathrm{Ala}^{4}-\mathrm{Val}^{5}-\mathrm{Leu}^{6}-\mathrm{Lys}^{7}-\mathrm{Val}^{8}{ }^{-\mathrm{Leu}^{9}}- \\
& \text { Thr }^{10}-\mathrm{Thr}^{11}-\mathrm{Gly}^{12}-\mathrm{Leu}^{13}-\mathrm{Pro}^{14}-\mathrm{Ala}^{15}-\mathrm{Leu}^{16}-\mathrm{Ile}^{17}-\mathrm{Ser}^{18}- \\
& \text { Trp }^{19}-\mathrm{Ile}^{20}-\mathrm{Lys}^{21}-\mathrm{Arg}^{22}-\mathrm{Lys}^{23}-\mathrm{Arg}^{24}-\mathrm{Gln}^{25}-\mathrm{Gln}^{26}
\end{aligned}
$$

Its structure has been determined to high-resolution by X-ray diffraction [18] and NMR spectroscopy. The NMR structure of melittin has been determined in methanol solution [19] and when bound to dodecylphosphocholine micelles [20, 21]. In both media, the 
peptide is monomeric [19, 22-24], whereas the crystals were grown from aqueous solution of high ionic strength, in which melittin forms tetrameric aggregates through hydrophobic contacts [13, 18, 25]. These crystallographic and NMR studies indicate that melittin adopts an $\alpha$-helical conformation in these rather different environments. The proline residue at position 14 causes a kink in the structure separating two $\alpha$-helical segments, a mostly hydrophobic helix from Gly(1) to Leu(13) (helix 1) and an amphiphilic, positively charged helix from Pro(14) to Gln(26) (helix 2) (Fig. 1). From high-resolution ${ }^{1} \mathrm{H}-\mathrm{NMR}$ experiments of monomeric melittin in aqueous solution it was concluded that the peptide does not adopt a regular structure in the absence of salt [26, 27].

With the aim to elucidate the mechanism of the lytic activity of melittin, the association of melittin with lipid bilayers and the initiation mechanisms of membrane lysis, the association of melittin with bilayers was investigated in a large number of studies using different approaches [12, 20, 22, 23, 28-39]. This led to mainly two views of how melittin interacts with lipid bilayers causing their lysis. On the one side, it is suggested that the peptide increases the permeability by binding parallel to the membrane surface with partial penetration of the bilayer, while on the other side experimental evidence has been put forward that melittin adopts a transmembrane orientation and forms water pores by aggregation of four peptide molecules. Yet, other studies suggest that melittin solubilises large lipid disks which would lead to cell lysis by leaving large holes in the membrane. The variety of proposed actions of melittin on membranes reflects the view that the underlying mechanism varies with a number of factors, such as peptide concentration, lipid composition, and the hydration level of the membrane [2, 38, 40].

In an attempt to describe the association of melittin with membranes and to elucidate the structural characteristics of melittin in different environments at the atomic level, a number of molecular dynamics (MD) simulation studies on melittin and also on other antimicrobial peptides have been performed. They have been reviewed La Rocca et al. [41] and by Biggin and Sansom [11]. The structural and hydrogen-bonding properties of melittin and alamethicin in methanol have been analysed from short (0.5-1 ns) molecular dynamics simulations and compared to the results from amide hydrogen exchange analysis [42]. More recently the conformational stability of melittin at different temperatures and in different solvent environments was investigated by short simulations (200 ps) using the the temperature jump technique [43] showing that low dielectric media stabilise the secondary structure of melittin. In a similar vein Roccatano et al. [44] have shown that the secondary structure of melittin is well preserved in 2,2,2-trifluoroethanol (TFE)/water mixtures over the entire $20 \mathrm{~ns}$ of simulation. The TFE molecules are observed to aggregate around the peptide forming a matrix that partly excludes water, and thus favours intramolecular, local interactions such as backbone-backbone hydrogen-bonding, which is in line with experimental findings from NMR studies [45]. In a series of simulations the effect of melittin on lipid bilayers was investigated when adsorbed to the membrane surface [46-49], inserted as a single transmembrane helix in a bilayer [50], and as a tetrameric water pore in a lipid bilayer [51]. When absorbed on the bilayer surface melittin is found to have an effect on both leaflets of the membrane. It causes thinning of the upper layer, which in turn favours water penetration through the lower layer [46]. Bachar and Becker [50] reported simulations of a single melittin molecule inserted in a dipalmitoylphosphatidylcholine (DPPC) bilayer in a transmembrane orientation and observed local disorder of the lipids in vicinity of the peptide. In the melittin pore simulation described by Lin and Baumgärtner [51], the individual helices in the tetrameric aggregate repelled each other due to their highly positively charged C-ter-
Fig. 1 a Crystallographic structure of melittin [18, 57] used as a starting structure in the simulations. b Melittin inserted in a DMPC bilayer (62 lipids per leaflet). The lipid headgroups are shown as spheres with the phosphorus atoms in orange, the oxygen atoms in yellow the nitrogen atoms in cyan and the $\mathrm{CH}_{3}$ groups in blue

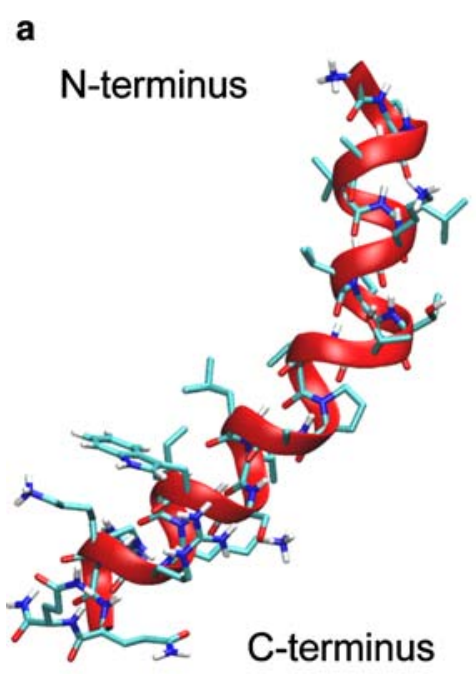

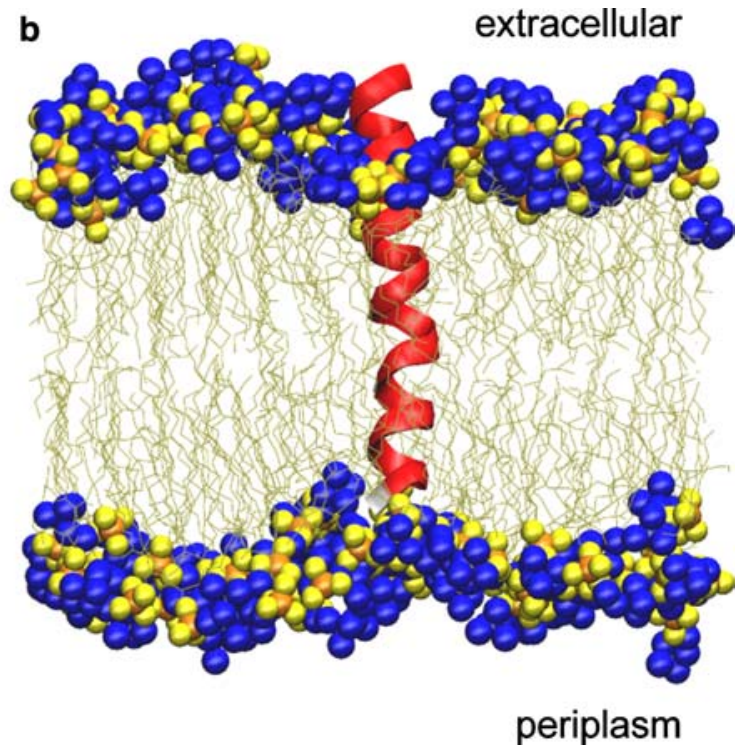


minus causing a disruption of the lipid bilayer within $4 \mathrm{~ns}$. Whether this represents a realistic description of the mechanism of cytolytic activity of melittin or is rather due to simulation artefacts is questionable.

The aim of the study presented here is not to elucidate by simulation the mechanisms of membrane lysis caused by melittin, but to investigate the structural properties of melittin in three different environments from which mechanistic insights may be derived. The peptide was simulated in aqueous solution, in methanol solution and as a transmembrane helix inserted in a dimyristoylphosphatidylcholine bilayer (Table 1, Fig. 1). Due to the different computational costs of simulating these three environments, the lengths of the three simulations are different: $30 \mathrm{~ns}$ in water, $60 \mathrm{~ns}$ in methanol, and $10+17 \mathrm{~ns}$ in the lipid bilayer. This should be kept in mind when interpreting the results. The simulation trajectories are analysed in terms of structural properties of the peptide such as secondary structure content and hydrogen bonding and with respect to the level of agreement with experimental NMR data $\left({ }^{3} \mathrm{~J}\right.$-coupling constants). In addition intermolecular hydrogen bonding between melittin and the surrounding lipids is investigated. The present study differs from earlier simulation studies of melittin with the respect to the force field used, the simulation set-up, comparison with NMR data and analysis of melittin-environment hydrogen bonding.

\section{Methods}

Table 1 gives an overview over the performed simulations. All simulations were carried out using the GROMOS96 simulation software $[52,53]$. The peptide was modeled using the GROMOS force field, version 45A3 $[52,54]$. The same force field under the conditions elucidated in reference [55] was used for the lipids, where the phosphatidylcholine (PC) head group parameters for the partial charges are taken from Chiu et al. [56]. The set-up of the protein-lipid systems, the simulation setup, and the type analysis performed are described here.
Molecular model

\section{Peptide model and solvent models}

The X-ray structure (PDB entry: 2MLT) [18, 57] was used as a starting structure for all four simulations. The ionisable groups were set to their protonated or deprotonated state according to the standard $\mathrm{pK}_{a}$ values of amino acids and a $\mathrm{pH}$ of 7.0. Consequently, the lysine and the arginine side-chains, as well as the N-terminus are protonated, resulting in a net charge of $+6 e$. The $\mathrm{C}$ terminus is amidated. Water and methanol were modelled as rigid three-point molecules using the SPC water model [58] and the standard GROMOS96 methanol model [52], respectively.

For simulations melittin-h2o and melittin-meoh, the peptide was solvated in a truncated-octahedron-shaped box. The size of the box was chosen such that the initial minimum distance between peptide atoms and the square walls of the truncated octahedron was $1.6 \mathrm{~nm}$. Periodic boundary conditions were applied. After an energy minimisation using steepest descent to relax the solvent, the six water molecules experiencing the largest positive Coulomb potential were replaced by six chlorine ions to neutralise the system.

\section{Peptide-bilayer model}

Melittin was inserted in a dimyristoyl-phosphatidylcholine (DMPC) bilayer, consisting of $2 \times 8 \times 8$ lipids. The hydrocarbon core of DMPC bilayers is measured to be $2.6 \mathrm{~nm}$ thick at room temperature [59], which is approximately comparable to the thickness of biological membranes. The pure bilayer (without peptide) was preequilibrated in a 5 ns simulation following the simulation protocol described in reference [55]. Subsequently, the peptide was placed in the middle of the bilayer and in each bilayer leaflet the two lipid molecules with the largest number of atom-atom overlaps with the peptide were removed. The total mass of the four removed lipids corresponds approximately to the mass of melittin. One

Table 1 Overview over the simulations performed

\begin{tabular}{|c|c|c|c|c|}
\hline & \multicolumn{4}{|l|}{ System/simulation name } \\
\hline & Melittin-h2o & Melittin-meoh & Melittin-dmpc-1 & Melittin-dmpc-2 \\
\hline Solvent & Water & Methanol & Water & Water \\
\hline Number of solvent molecules & 5,465 & 2,430 & 4,884 & 4,738 \\
\hline Number of lipids & - & - & 124 & 124 \\
\hline number of counter-ions & $6 \mathrm{Cl}^{-}$ & $6 \mathrm{Cl}^{-}$ & $6 \mathrm{Cl}^{-}$ & $6 \mathrm{Cl}^{-}$ \\
\hline Total number of atoms & 16,663 & 7,558 & 20,624 & 20,186 \\
\hline Type of box & Truncated octahedron & Truncated octahedron & Rectangular & Rectangular \\
\hline Box lengths (nm) & $6.9-6.9-6.9$ & $6.9-6.9-6.9$ & $5.8 \times 6.2 \times 8.0$ & $5.6 \times 6.2 \times 8.0$ \\
\hline Equilibration time (ns) & 0.1 & 0.1 & 0.2 & 1.6 \\
\hline Simulation (production) time (ns) & 30 & 60 & 10 & 17 \\
\hline
\end{tabular}

For the four simulations, melittin-h2o, melittin-meoh, melittin-dmpc-1, and melittin-dmpc-2, the most important simulation details are given. All four simulations were started from the crystal-structure (PDB entry: 2MLT) [18, 57]. Simulation melittin-dmpc-2 was started from the configuration after $1.5 \mathrm{~ns}$ of simulating melittin-dmpc-1 after readjusting the helix axis parallel to the $z$-axis of the membrane (see text for details). The box lengths for the truncated octahedron are indicated as the distances between the square planes 
of the ubiquitous features of membrane protein and peptides is that aromatic residues (Trp, Tyr in particular) interact with the hydrophobic-hydrophilic interface of membranes [60]. In addition, from fluorescence quenching measurements on melittin it was concluded that the C-terminus of melittin remains at the outer surface of the membrane, i.e. at the extracellular side [61, 28]. Accordingly, the melittin was inserted in the membrane with the C-terminus at the upper (extracellular) side of the membrane (Fig. 1b) such that the $\operatorname{Trp}(19)$ is approximately at the average z-position of the $\mathrm{C} 2$-atoms of the DMPC lipids.

The resulting peptide-bilayer system was energy minimised to remove the remaining bad lipid-protein contacts using the steepest descent method while keeping the peptide atoms positionally restrained. The system was then solvated in a (periodic) box using a pre-equilibrated box of SPC water. All water molecules that were placed on the lipid phase in the process of solvation were subsequently removed resulting in a system size of more than 20000 atoms (Table 1). The water was relaxed in a further energy minimisation with the lipids and the peptide positionally restrained (force constant $2.5 \times 10^{-4} \mathrm{~kJ} \mathrm{~mol}^{-1} \mathrm{~nm}^{-2}$ ) and in a first equilibration simulation of $100 \mathrm{ps}$ with only the peptide restrained following the simulation protocol outlined below. Thereafter six randomly chosen water molecules were replaced by six chlorine ions to neutralise the system and a third energy minimisation was performed with the peptide positionally restrained.

The simulation melittin-dmpc-2 was started from the conformation obtained after $1.5 \mathrm{~ns}$ of simulating melittin-dmpc-1, realigning the peptide in parallel to the bilayer normal. The same set-up and equilibration procedure was applied as for simulation melittin-dmpc1. The reason for this second simulation starting with a reoriented peptide is the following: for simulation melittin-dmpc-1 the transmembrane orientation, parallel to the membrane normal, of the initial melittin helix, the Xray structure, was conducted with respect to helix 2 . In the course of simulation it was observed that the helical kink became less pronounced and the structure adopted a diagonal orientation within the membrane. In order to test whether this is an artefact resulting from the fact that the initial X-ray structure does not necessarily need to be identical to the structure of melittin in a membrane, melittin was reoriented in simulation melittindmpc-2.

\section{Simulation protocol}

The MD simulations were started by taking the initial velocities from Maxwellian distributions at $50 \mathrm{~K}$ for the melittin in solution and at $300 \mathrm{~K}$ for the bilayer systems. Solvent and solute (peptide or peptide-lipid system) were independently coupled to a temperature bath with a relaxation time of 0.1 ps [62]. The pressure was calculated with a molecular virial and held constant by weak coupling to a pressure bath with a relaxation time of $0.5 \mathrm{ps}$ and using an isothermal compressibility of $4.575 \times 10^{-4}\left(\mathrm{~kJ} \mathrm{~mol}^{-1} \mathrm{~nm}^{-3}\right)^{-1}$. Note that for all the equilibration simulations where the protein was positionally restrained, the simulations were performed at constant volume (NVT). Thus no pressure coupling was applied under these conditions. Bond lengths were constrained using the SHAKE algorithm [63] with a geometric tolerance of $10^{-4}$. The equations of motion were integrated using the leap-frog algorithm and a time step of $2 \mathrm{fs}$. The interaction between atoms in so-called charge groups [52] was calculated according to a spherical triple-range cutoff scheme: short-range van der Waals and electrostatic interactions were evaluated at every time step by using a charge-group pair list that was generated with a short-range cutoff radius of $0.8 \mathrm{~nm}$ between the centers of geometry of the charge groups. Long-range van der Waals and electrostatic interactions, between pairs at a distance greater than $0.8 \mathrm{~nm}$ and shorter than a long-range cutoff of $1.4 \mathrm{~nm}$, were evaluated every fifth time step, at which point the pair list was also updated, and were kept unchanged between these updates. To approximate the electrostatic interactions beyond the long-range cutoff, a Poisson-Boltzmann reaction-field force was used. In simulation melittin-h2o and melittin-meoh the value for the dielectric permittivity of the continuum outside the long-range cutoff was set to 62 and 32, respectively. For the peptide-bilayer this value was set to 54, following the protocol standardly used for lipid bilayer simulations [55].

For the two simulations of melittin in solution, the equilibration process was as follows. After addition of the counter-ions, the system was equilibrated for 80 ps during which the temperature was stepwise increased to $300 \mathrm{~K}$ and the restraining force decreased until the atoms of the peptide were unrestrained. After this initial equilibration, the pressure coupling was switched on and a further equilibration simulation of $120 \mathrm{ps}$ was performed before entering the production phase.

For the two simulations of melittin in a DMPC bilayer, the atoms of the peptide were positionally restrained using a harmonic restraining force with a force constant of $2.5 \times 10^{4} \mathrm{~kJ} \mathrm{~mol}^{-1} \mathrm{~nm}^{-2}$ during the $110 \mathrm{ps}$ of equilibration before adding the counter-ions. After the addition of counter-ions as described above, the system was further equilibrated for $110 \mathrm{ps,} \mathrm{while} \mathrm{the} \mathrm{force}$ constant of the restraining force was reduced step-wise to zero. At this point the pressure coupling was switched on and another equilibration period of 100 ps was added before saving configurations for analysis. This results in a total equilibration time of 320 ps.

\section{Analysis}

The analyses were performed on the ensemble of system configurations extracted at $0.5 \mathrm{ps}$ time intervals from the simulations. Least-squares fitting of atomic coordinates for the calculation of structural properties of the peptide 
Fig. 2 Atom-positional rootmean-square deviation ( $r m s d$ ) of melittin with respect to the initial X-ray structure for the simulations melittin-h2o (a), melittin-meoh (b), melittindmpc-1 (c), and melittin-dmpc2 (d). The different lines show the rmsd of the backbone atoms $\left(\mathrm{N}, \mathrm{C}_{\alpha}, \mathrm{C}\right)$ of residues $2-25$ (black), of the backbone atoms of helix 1 (residues 2-13, grey), and of the backbone atoms of helix 2 (residues 14-25, lightgrey). For the simulation melittin-dmpc-2 the rmsd of the backbone atoms of residues $2-$ 25 from the conformation obtained after $1.5 \mathrm{~ns}$ of simulation melittin-dmpc-1, from which the second simulation in a DMPC bilayer has been started, is additionally shown (drak-grey line in $\mathrm{d}$ ). Note that the time scale differs between the upper and the lower panels
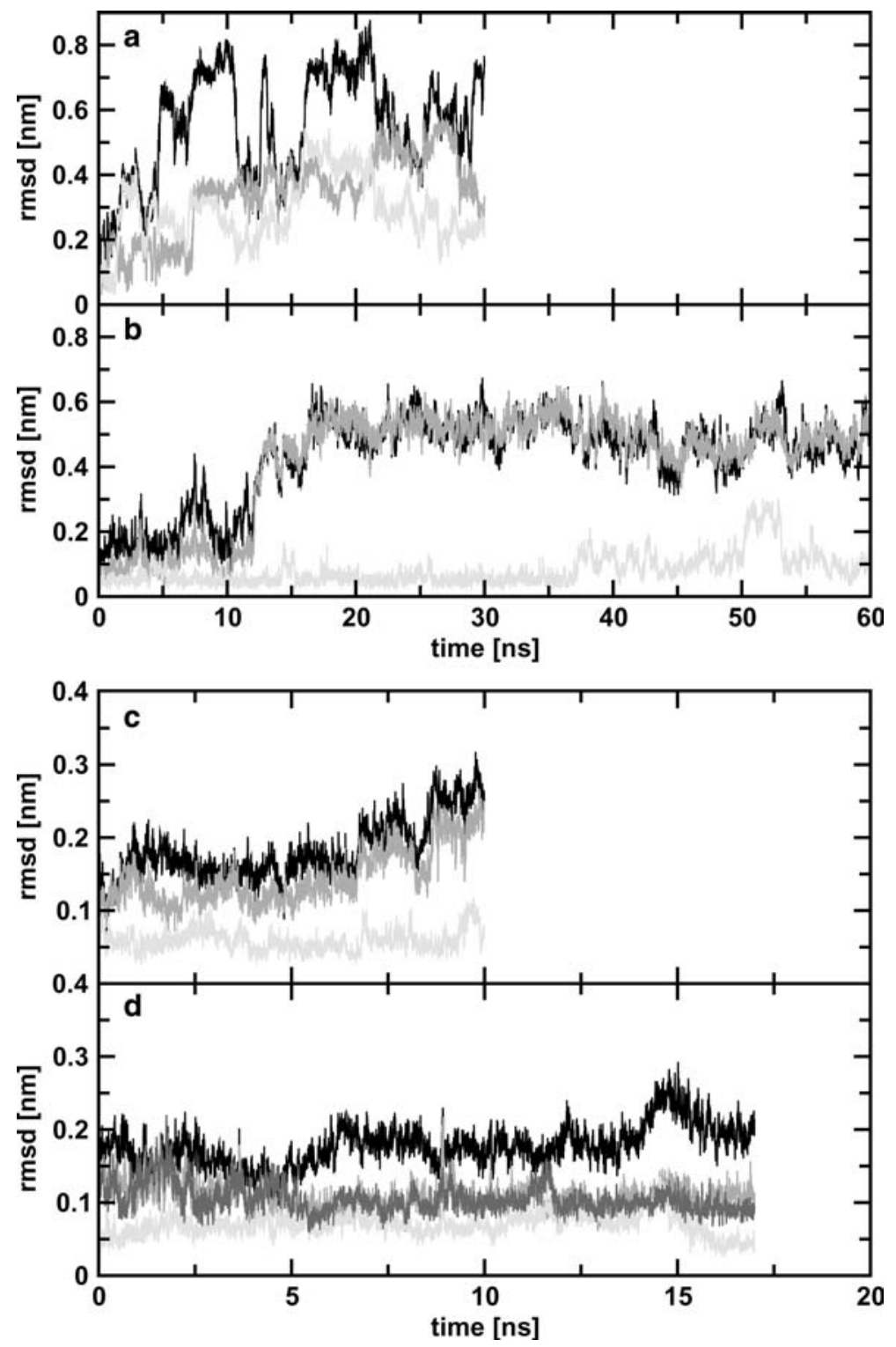

such as the atom-positional root-mean-square deviation or difference (rmsd) was based on the backbone atoms $(\mathrm{N}, \mathrm{C} \alpha, \mathrm{C})$ of all residues but the first and the last one (residues 2-25). For the calculation of the rmsd of the first helix the backbone atoms of residues 2-13 were used and for the rmsd of the second helix the backbone atoms of residues 14-25 were employed. The root-meansquare fluctuations (rmsf) were calculated for the $\mathrm{C} \alpha$ atoms.

The helical kink induced by the proline residue at position 14 in melittin was measured by calculating the intersecting angle of the helical axis of helix 1 (residues 1-13) and helix 2 (residues 14-26). To determine the axis of a helix the "Kahn" method described by Christopher et al. [64] was applied. This approach uses the fact that the angle between three consecutive, evenly spaced atoms is bisected by a vector, perpendicular to the helical axis. From the cross-product of two such vectors the helical axis can be determined.

The secondary structure assignment of melittin for the set structures extracted every 50 ps from the simulation trajectories was done according to the DSSP rules proposed by Kabsch and Sander [65]. The hydrogen bonds were calculated using a geometric criterion. A hydrogen bond is defined by a minimum donor-hydrogen-acceptor angle of $135^{\circ}$ and a maximum hydrogenacceptor distance of $0.25 \mathrm{~nm}$.

${ }^{3} J$-coupling constants were calculated from the simulation using the Karplus relation [66],

${ }^{3} \mathrm{~J}(\mathrm{H}, \mathrm{H})=a \cos ^{2} \theta+b \cos \theta+c$,

where $a, b$ and $c$ were chosen equal to $6.4,-1.4$, and $1.9 \mathrm{~Hz}$, respectively, for the calculation of ${ }^{3} \mathrm{~J}(\mathrm{HN}, \mathrm{HC})$ [67]. 
Fig. 3 Helix kink of melittin induced by the proline residue in position 14 as a function of simulation time. The proline kink corresponds to the intersection angle between the two helical axis of helix 1 (residues 1-13) and helix 2 (residues 14-26) following the method described by Christopher et al. [64]. The dashed line corresponds to the proline kink calculated from the $\mathrm{X}$-ray structure [18, 57]. a Simulation melittin-dmpc-1; b Simulation melittin-dmpc-2

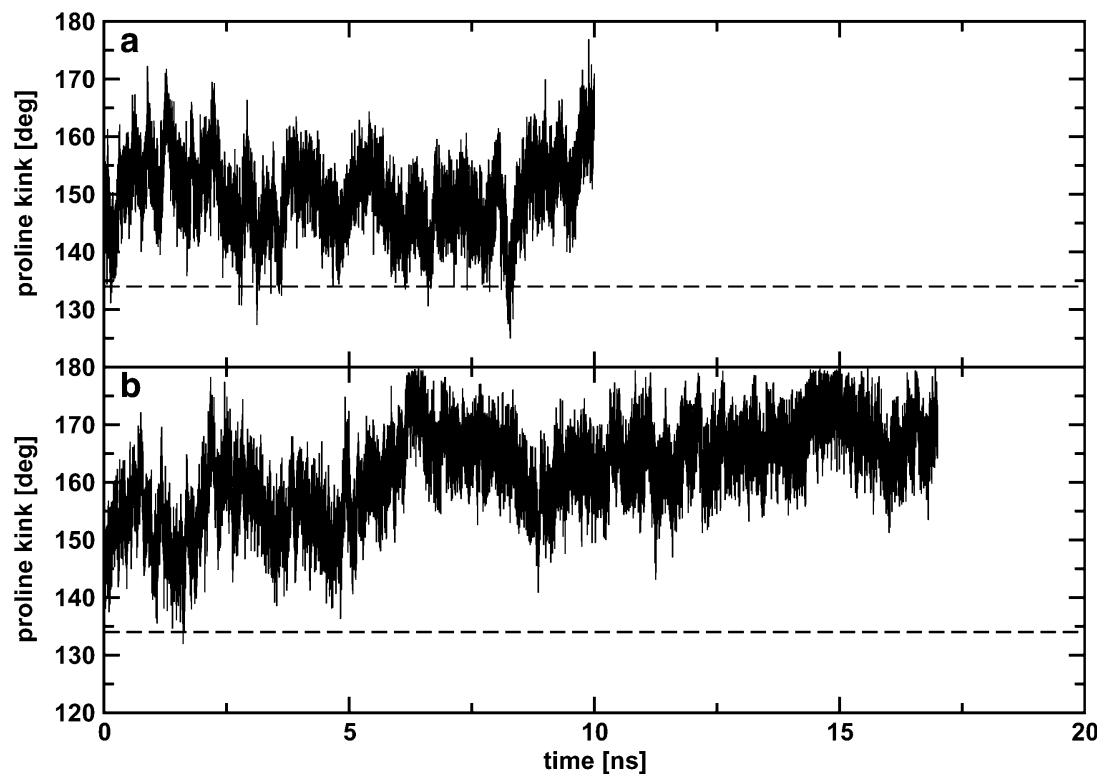

\section{Results and discussion}

Figure 2 shows the rmsd from the crystal structure[18], from which all four simulations have been started, as a function of time for the simulations melittin-h2o (Fig. 2a), melittin-meoh (Fig. 2b), melittin-dmpc-1 (Fig. 2c) and melittin-dmpc-2 (panel D). The black lines correspond to the rmsd of the entire peptide (residues 225 ), the grey lines correspond to the rmsd of helix 1 (residues 2-13) before the proline-kink, and the lightgrey lines show the rmsd of helix 2 (residues 14-25), after the proline-kink. For the simulation melittin-dmpc-2, the rmsd calculated from the melittin structure used to start the melittin-dmpc-2 simulation (dark-grey line in panel C of Fig. 2) was additionally calculated. There is a clear difference in the stability of the structure of melittin between the solution and the bilayer simulations. In water, the peptide rapidly unfolds. Both helices show relatively large rmsd-values with respect to the X-ray structure, although the positively charged helix 2 (light grey line) seems to partly refold during the last $10 \mathrm{~ns}$ of simulation. In methanol, the overall structure appears to be stable for the first $10 \mathrm{~ns}$ of simulation, after which the helix is observed to unfold. The rmsd values for the individual helices show that helix 1 (grey line) unfolds and essentially remains unfolded for the remaining $50 \mathrm{~ns}$ of simulation, while helix 2 (light-grey line) remains stable over the entire $60 \mathrm{~ns}$ of simulation. When embedded in a lipid bilayer melittin appears to be more stable. In both simulations, melittin-dmpc-1 and melittin-dmpc-2, the rmsdeviation from the X-ray structure is low compared to the simulations in solution $(<0.3 \mathrm{~nm}$ for melittin-dmpc1 and less than $0.25 \mathrm{~nm}$ for melittin-dmpc-2). Helix 2 (light-gray line) appears to stay closer to the helical conformation than helix 1 (grey line), which is a comparable to the observation made in the simulations in solution. In simulation melittin-dmpc-2, the rmsd from the X-ray structure is on average $0.07 \mathrm{~nm}$ larger than the rmsd from the melittin structure the simulation has been started from. This is probably due to the more pronounced helix kink of the X-ray structure compared to the melittin-dmpc-2 starting structure (see Fig. 3). The latter corresponds to the conformation obtained after 1.5 ns of simulation melittin-dmpc-1 (see Methods section for details). The proline kink of melittin appears to be less pronounced in a lipid bilayer environment than found in the X-ray structure of the tetrameric aggregate. In simulation melittin-dmpc-2 the relative orientation of the two helices (helix 1: residues 1-13 and helix 2: residues 14-26) seems to converge close to $180^{\circ}$.

The secondary structure assignment according to the rules by Kabsch and Sander [65] presented in Fig. 4 and in Table 2 confirm the conclusions drawn from the atom-positional rmsd analysis. In water (Fig. 4a-1) the overall structure of the peptide is relatively unstable, while in methanol (Fig. 4b-1) unfolding is only observed from the N-terminus to Pro-13. Helix 2 (residues 14-26) shows a higher stability in both solvent environments than the hydrophobic helix 1. When inserted in a DMPC bilayer, the secondary structure of melittin is well preserved over the entire simulation (Fig. 4c1-c2) showing an average $\alpha$-helical content of 86 and $84 \%$ (Table 2). According to the atom-positional rms fluctuation and the varying degree of secondary structure conservation in the simulations, it is clear that the peptide shows a larger structural mobility when solvated in water or methanol than as a transmembrane helix in a DMPC bilayer (Fig. 4a-2-d-2).

Figure 5 shows the final melittin conformation of each of the four simulations and gives an impression of the different behaviour of melittin in aqueous solution, in methanol and in the DMPC membrane. In water 
(Fig. 5a) the peptide has collapsed burying some of the hydrophobic residues with part of the $\alpha$-helical structure intact. In methanol (Fig. 5b) the N-terminal part is practically extended, while the secondary structure of helix 2 is retained. In order to compare the compactness of the structures in the two different solvent environments over the entire simulation, the radius of gyration of the peptide is presented as a function of time for the simulations melittin-h2o and melittin-meoh in Fig. 6. In water, the radius of gyration fluctuates between the initial value of 1.2 and $0.8 \mathrm{~nm}$ (black line in Fig. 6), while in methanol, the radius of gyration is observed to increase after $10 \mathrm{~ns}$ from 1.2 up to $1.6 \mathrm{~nm}$ (grey-line in Fig. 6) reflecting the more extended conformation of the $\mathrm{N}$-terminal part of melittin in methanol. In stark contrast, the structures in the DMPC bilayer retain their $\alpha$-helical character. Consistent with the rms deviation shown in Fig. 2, helix 2 (residues 14-26) is more intact than helix 1 (residues 1-13). The relative orientation of the helices appears to vary slightly between the two simulations (melittin-dmpc-1 and melittin-dmpc-2) as also illustrated by the difference in helix tilt in Fig. 3).

The analysis of the intramolecular backbone hydrogen bonds basically reflects the observations made in the conformational analysis of the simulations (see Table 3). Hydrogen bonds characteristic for the $\alpha$-helical conformation are largely populated in the simulations melittindmpc-1 and melittin-dmpc-2, while in methanol only residues 12-24 show large hydrogen-bond populations of more than $70 \%$. In water, the $\alpha$-helical hydrogen bonds appear to be less populated in this region of the peptide, while the hydrogen-bond populations in the
Fig. 4 Left-hand panels secondary structure of melittin as a function of time in the simulations melittin-h2o (a-1), melittin-meoh (b-1), melittindmpc-1 (c-1), and melittindmpc-2 (d-1). Red $\alpha$-helix; yellow $3{ }_{10}$-helix; orange $\pi$-helix; blue bend; green extended $\beta$ strand; violet turn; according to the definitions of secondary structure by Kabsch and Sander [65]. Right-hand panels Atompositional root-mean-square fluctuation (rmsf) of the $\mathrm{C}_{\alpha-}$ atoms as derived from the simulations melittin-h2o (a-2), melittin-meoh (b-2), melittindmpc-1 (c-2), and melittindmpc-2 (d-2). Note that the time scale differs between the upper and lower panels

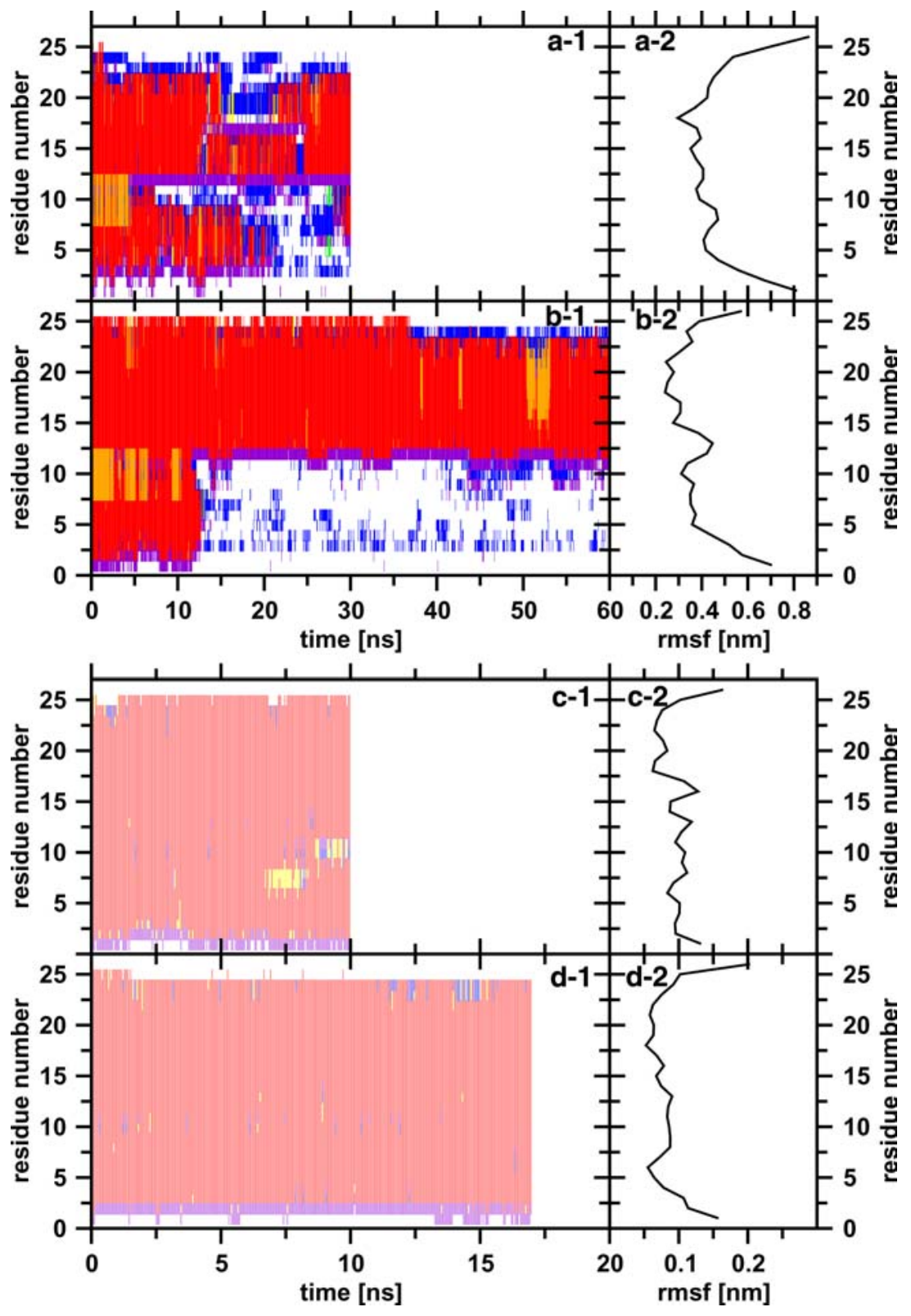


Table 2 Secondary structure content in melittin

Secondary structure content in melittin $(\%)$

\begin{tabular}{llllll}
\hline Secondary structure element & X-ray & Melittin-h2o & Melittin-meoh & Melittin-dmpc-1 & Melittin-dmpc-2 \\
\hline$\alpha$-Helix & 92 & 42 & 51 & 86 & 84 \\
$3_{10}$-Helix & - & 2 & - & 2 & 1 \\
$\pi$-Helix & - & 3 & 3 & - & - \\
Bend & - & 15 & 6 & 5 & 1 \\
Turn & 4 & 9 & 35 & 6 & 4 \\
"Random coil" & 4 & 29 & & 10
\end{tabular}

The Table indicates the percentage of amino acid residues to which a certain secondary structure element has been assigned according to the rules defined by Kabsch and Sander [65] in the Xray structure and in the structures extracted at 50 ps intervals from the four simulations melittin-h2o, melittin-meoh, melittin-dmpc-1, melittin-dmpc-2

Fig. 5 Final conformations of melittin in the simulations melittin-h2o (a, $30 \mathrm{~ns})$, melittinmeoh (b, 60 ns), melittin-dmpc1 (c, $10 \mathrm{~ns})$, and melittin-dmpc$2(\mathbf{d}, 17 \mathrm{~ns})$ a

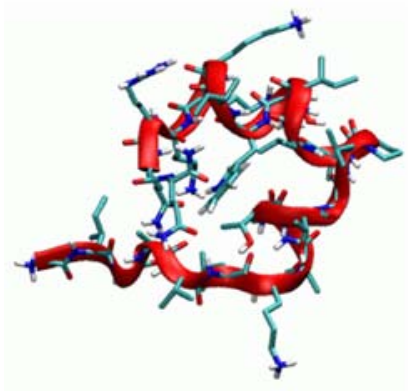

\section{C-terminus}

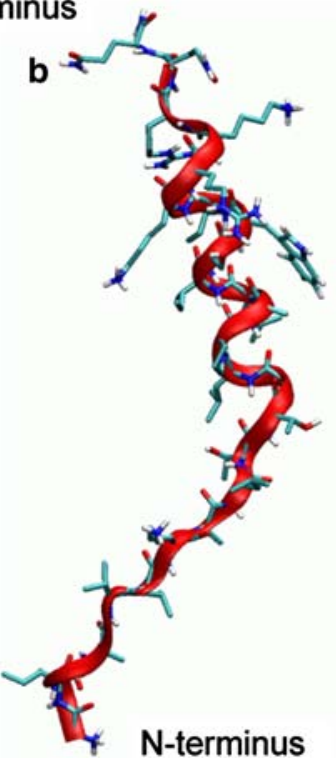

C

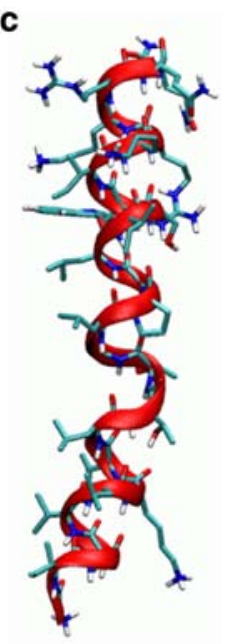

d

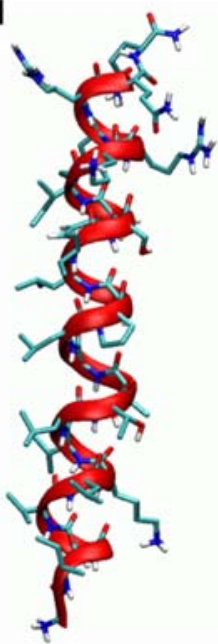

Fig. 6 Radius of gyration of melittin as a function of time for the simulation melittin-h2o (black) and melittin-meoh (grey).

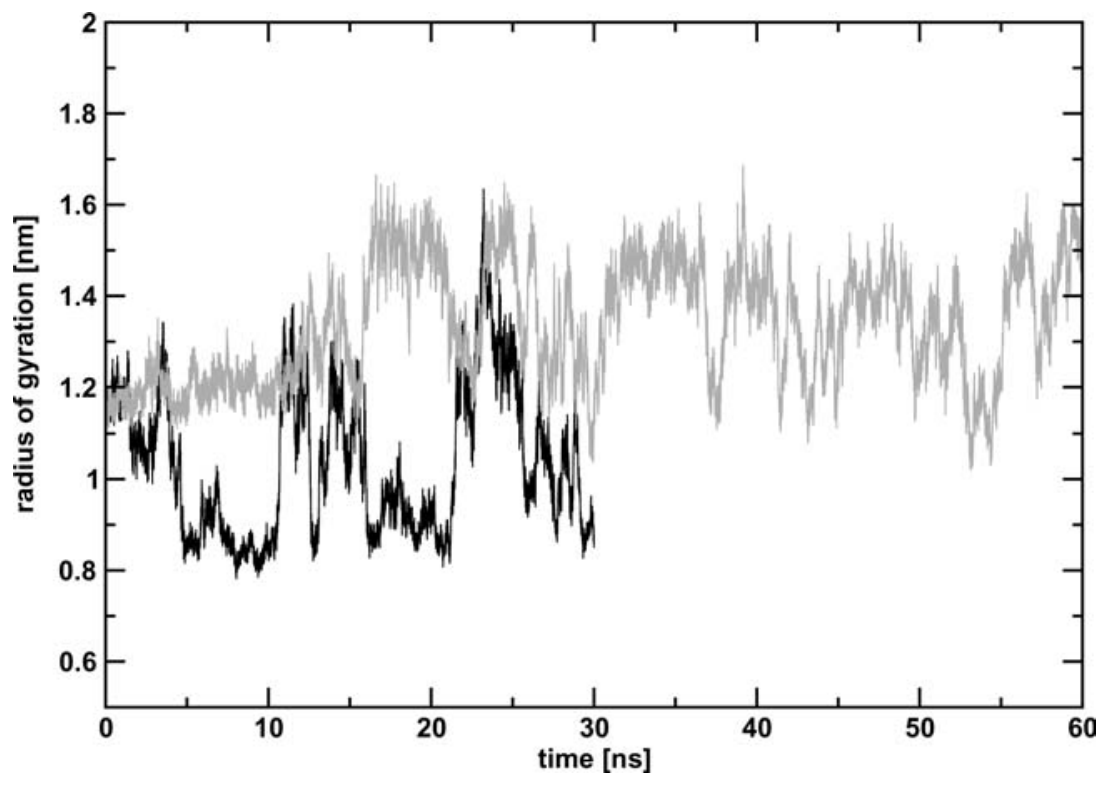


Table 3 Intramolecular hydrogen-bond populations

Intramolecular backbone-backbone hydrogen bond populations (\%)

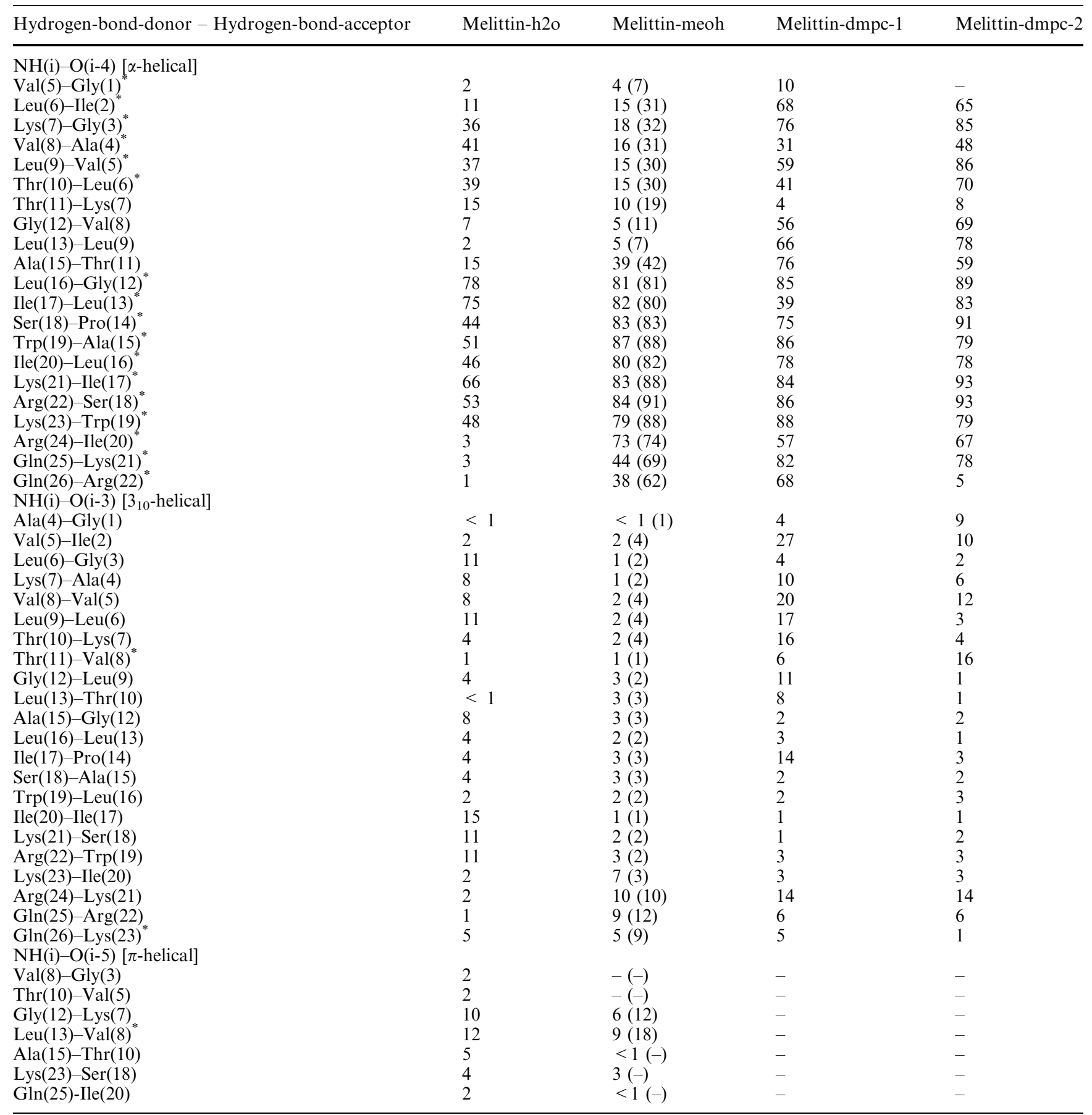

Only backbone-backbone hydrogen bonds with a population larger than $2 \%$ in at least one of the simulations are shown. A hydrogen bond is considered to exist when the donor-hydrogen-acceptor angle is larger than $135^{\circ}$ and the hydrogen-acceptor distance is smaller than $0.25 \mathrm{~nm}$. The hydrogen bonds are grouped according the secondary structure element they are characteristic of $\left(\alpha\right.$-helix, $3_{10}$-helix, $\pi$ helix). The hydrogen bonds marked with an asterix correspond to hydrogen bonds detected in the crystallographic structure. The values between parentheses for the simulation melittin-meoh correspond to hydrogen-bond populations averaged over the first $30 \mathrm{~ns}$ of the simulation

region of helix 1 are larger than in the simulation in methanol. Apart from the hydrogen bonds typical for $\alpha$ helices, hydrogen bonds characteristic for $3_{10}$-helical and $\pi$-helical conformations are observed. In solution, both types occur at populations up to $15 \%$, while melittin inserted in a lipid bilayer only exhibits $310^{\text {-helical }}$ hydrogen bonds. Recently it has been discussed that the sampling of $\pi$-helical conformations, which are rather 
Table $4{ }^{3} \mathrm{~J}(\mathrm{NH}-\mathrm{C} \alpha \mathrm{H})$-coupling constants for melittin in methanol extracted from the ${ }^{1} \mathrm{H}-\mathrm{NMR}$ spectrum $(\mathrm{CD} 3 \mathrm{OH}$, 500 MHz, room temperature) [19] and calculated for all recorded structures in the simulations melittin-h2o, melittin-meoh, melittin-dmpc-1, and melittindmpc-2 according to the Karplus relation [66], using $a=6.4 \mathrm{~Hz}, b=-1.4 \mathrm{~Hz}$, and $c=1.9 \mathrm{~Hz}$ [67].

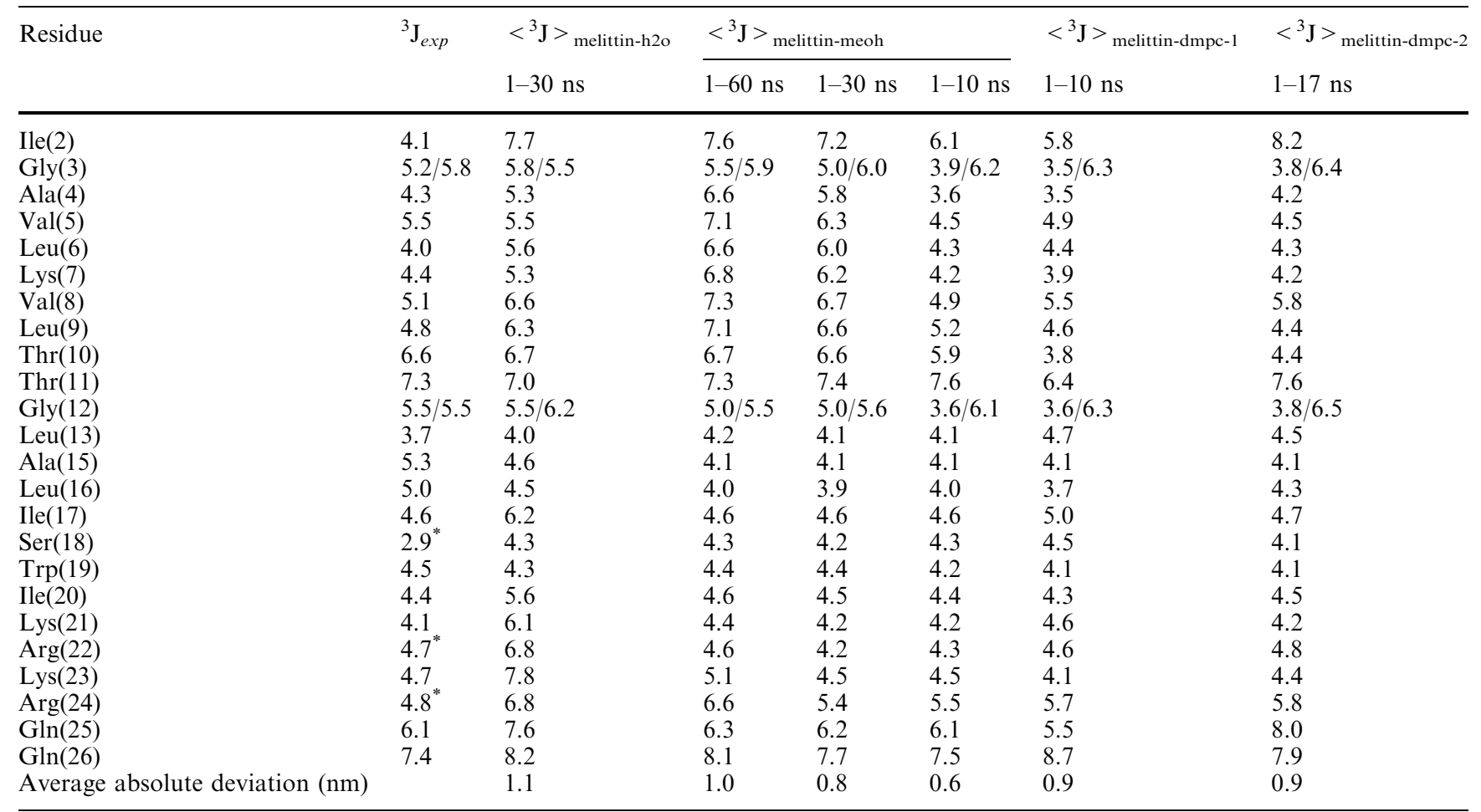

For simulation melittin-meoh the ${ }^{3} \mathrm{~J}$-coupling constants are also calculated over the first $10 \mathrm{~ns}$, where the full $\alpha$-helix was retained, and over the first $30 \mathrm{~ns}$ of the simulation for ease of comparison with the simulation melittin-h2o. The experimental ${ }^{3} \mathrm{~J}$-values marked with an asterix are less precise due to overlaps which could not be resolved [19]

rarely observed in X-ray structures of peptides and proteins, may be an artefact of empirical biomolecular force fields [68]. Although we observe part of melittin to adopt a $\pi$-helical conformation for short periods of time, no overall transition from an $\alpha$-helix to a $\pi$-helix is observed in the simulations presented here in contrast to other MD studies [68, 69].

In Table 4 the ${ }^{3} \mathbf{J}$-coupling constants calculated from the simulation trajectories are compared to the values extracted from the ${ }^{1} \mathrm{H}-\mathrm{NMR}$ spectrum in methanol solution [19]. There is good agreement between the simulated ${ }^{3} \mathrm{~J}$-coupling constants and the experimental values for the simulations melittin-dmpc-1 and melittindmpc-2. For the simulation in water and in methanol, averaged over the 30 or $60 \mathrm{~ns}$ of simulation respectively, the deviations from the experimental values are rather large in the region of helix 1 , the part of melittin that is observed to unfold in both simulations. Certainly, the ${ }^{3} \mathrm{~J}$-coupling constants averaged over the first $10 \mathrm{~ns}$ of the simulation in methanol where the full $\alpha$-helix was retained show a high level of agreement with the experimental values. The average absolute deviation of the calculated ${ }^{3} \mathrm{~J}$-coupling constants from experiment lies between $0.6 \mathrm{~Hz}$, for the average over the first $10 \mathrm{~ns}$ of the methanol simulation, and $1.1 \mathrm{~Hz}$ for the simulation in water. A variation of $1 \mathrm{~Hz}$ was observed when cal- culating ${ }^{3} \mathrm{~J}\left(\mathrm{NH}, \mathrm{C}_{\alpha} \mathrm{H}\right)$ values from one set of molecular structures using four different sets of commonly used parameters $a, b$ and $c$ for the Karplus relation [70]. This indicates that the (dis)agreement obtained here between simulation and experiment is satisfactory, considering the empirical nature of the Karplus parameters.

Summarising, the simulations presented here show that the helical conformation of melittin appears to be very stable when inserted in lipid bilayer in a transmembrane orientation, while in solution, either in water or in methanol, the peptide is observed to unfold and to loose part of its secondary structure. Generally helix 2 (residues 14-26) appears to be more stable than helix 1 (residues 1-13). Especially in methanol solution, helix 2 shows a remarkable stability over the entire $60 \mathrm{~ns}$ of simulation. The observation that only part of the $\alpha$-helical conformation in melittin is stable in methanol stands in contrast to the results from NMR studies reporting an overall $\alpha$-helical conformation in methanol [19, 24, 71]. Apart from the possibility that the force field is not able to correctly reproduce the conformational distribution of melittin in methanol, the N-terminal part of the peptide might eventually refold when extending the simulation to longer timescales. It is certainly more probable for the peptide to refold in methanol than in water considering that the 
Table 5 Intermolecular peptide-lipid hydrogen-bond populations

Intermolecular peptide-lipid hydrogen bond populations [\%]

\begin{tabular}{|c|c|c|}
\hline HB-donor - HB-acceptor & Melittin-dmpc-1 & Melittin-dmpc-2 \\
\hline Gly(1)-NH-DMPC(19)-O12 & 39 & \\
\hline Gly(1)-NH-DMPC(23)-O12 & 47 & \\
\hline Ile(2)-NH-DMPC(25)-O22 & 21 & \\
\hline $\mathrm{Ile}(2)-\mathrm{NH}-\mathrm{DMPC}(29)-\mathrm{O} 33$ & & 22 \\
\hline Gly(3)-NH-DMPC(29)-O33 & & 17 \\
\hline Val(5)-NH-DMPC(23)-O34 & & 49 \\
\hline Lys(7)- $\mathrm{N}_{\zeta} \mathrm{H}-\mathrm{DMPC}(13)-\mathrm{O} 12$ & & 45 \\
\hline Lys(7)-N $\mathrm{H}-\mathrm{DMPC}(23)-\mathrm{O} 22$ & 69 & \\
\hline Lys $(7)-\mathrm{N}_{\zeta}^{5} \mathrm{H}-\mathrm{DMPC}(26)-\mathrm{O} 22$ & 75 & \\
\hline Lys(7)-N $\mathrm{N}_{5}^{5} \mathrm{H}-\mathrm{DMPC}(26)-\mathrm{O} 21$ & & 42 \\
\hline $\mathrm{Thr}(11)-\mathrm{O}_{\gamma 1} \mathrm{H}-\mathrm{DMPC}(93)-\mathrm{O} 12$ & 28 & \\
\hline Ser(18)-O $\mathrm{H}-\mathrm{DMPC}(93)-\mathrm{O} 22$ & 89 & \\
\hline Ser(18)-O ${ }_{\gamma} \mathrm{H}-\mathrm{DMPC}(93)-\mathrm{O} 12$ & & 98 \\
\hline Trp(19)-N ${ }_{\varepsilon 1} \mathrm{H}-\mathrm{DMPC}(83)-\mathrm{O} 33$ & 38 & \\
\hline $\operatorname{Trp}(19)-\mathrm{N}_{\varepsilon 1} \mathrm{H}-\mathrm{DMPC}(112)-\mathrm{O} 22$ & & 20 \\
\hline Lys(21)-N $\mathrm{N}_{\zeta} \mathrm{H}-\mathrm{DMPC}(85)-\mathrm{O} 22$ & & 28 \\
\hline Lys(21)-N & & 59 \\
\hline $\operatorname{Arg}(22)-\mathrm{N}_{\eta 1} \mathrm{H}-\mathrm{DMPC}(72)-\mathrm{O} 32$ & 15 & \\
\hline $\operatorname{Arg}(22)-\mathrm{N}_{\eta 1} \mathrm{H}-\mathrm{DMPC}(72)-\mathrm{O} 33$ & 20 & \\
\hline $\operatorname{Arg}(22)-\mathrm{N}_{\eta 1} \mathrm{H}-\mathrm{DMPC}(87)-\mathrm{O} 12$ & 26 & \\
\hline $\operatorname{Arg}(22)-\mathrm{N}_{\eta 1} \mathrm{H}-\mathrm{DMPC}(93)-\mathrm{O} 22$ & 73 & \\
\hline $\operatorname{Arg}(22)-\mathrm{N}_{\eta 2} \mathrm{H}-\mathrm{DMPC}(93)-\mathrm{O} 22$ & 64 & \\
\hline $\operatorname{Arg}(22)-\mathrm{N}_{\varepsilon} \mathrm{H}-\mathrm{DMPC}(87)-\mathrm{O} 22$ & & 20 \\
\hline $\operatorname{Arg}(22)-\mathrm{N}_{\eta 2} \mathrm{H}-\mathrm{DMPC}(87)-\mathrm{O} 22$ & & 44 \\
\hline Lys(23)-N $\mathrm{H}-\mathrm{DMPC}(112)-\mathrm{O} 22$ & & 45 \\
\hline $\mathrm{Gln}(25)-\mathrm{N}_{\varepsilon 2}^{5} \mathrm{H}-\mathrm{DMPC}(72)-\mathrm{O} 34$ & 14 & \\
\hline $\mathrm{G} \ln (25)-\mathrm{N}_{\varepsilon 2} \mathrm{H}-\mathrm{DMPC}(77)-\mathrm{O} 33$ & 13 & \\
\hline $\mathrm{Gln}(25)-\mathrm{N}_{\varepsilon 2} \mathrm{H}-\mathrm{DMPC}(87)-\mathrm{O} 34$ & 14 & \\
\hline $\mathrm{G} \ln (25)-\mathrm{N}_{\varepsilon 2} \mathrm{H}-\mathrm{DMPC}(87)-\mathrm{O} 33$ & 44 & \\
\hline $\mathrm{G} \ln (25)-\mathrm{N}_{\varepsilon 2} \mathrm{H}-\mathrm{DMPC}(87)-\mathrm{O} 31$ & 18 & \\
\hline Gln(26)-NH-DMPC(86)-O12 & & 16 \\
\hline $\mathrm{G} \ln (26)-\mathrm{N}_{\varepsilon 2} \mathrm{H}-\mathrm{DMPC}(93)-\mathrm{O} 34$ & & 28 \\
\hline
\end{tabular}

Only hydrogen bonds with a population larger than $10 \%$ in at least one of the simulations are shown. The sequence numbers of the DMPC molecules are given between parentheses, the (standard) atom nomenclature for DMPC is according to reference 55. For more information see caption of Table 3

$\mathrm{N}$-terminus in the methanol solution is observed to be mainly extended, while in water the peptide showed a hydrophobic collapse (Figs. 5 and 6). On the other hand, it is not surprising that melittin partly unfolds in solution as it contains a proline residue in the middle. Proline is considered to be the classic helix breaker [72]. Due to its inability to act as a backbone hydrogen bond donor, it can only be tolerated in the three $\mathrm{N}$ terminal positions of $\alpha$-helices. Not surprisingly, only a handful of $\alpha$-helices containing internal proline residues are found in globular, water soluble proteins [73]. However, in apolar or amphiphilic transmembrane, helices of integral membrane proteins proline is frequently found in the middle of a TM-helix [74, 75]. The cis-trans isomerization of proline was suggested to play a key role in transport membrane proteins $[76,74,77]$. The experimental evidence that helices with a proline residue in the middle would partly be unfolded in solution, but would retain their full $\alpha$-helicity as a transmembrane helix embedded in a lipid bilayer supports the observations made in the simulations presented here. The increased stability of melittin inserted in a DMPC bilayer is certainly due to the absence of competing hydrogen bond donors and acceptors and is also reflected by the less pronounced helix tilt of melittin when inserted in a DMPC bilayer (Fig. 3).

The investigation of intermolecular peptide-lipid hydrogen bonds, presented in Table 5, gives partial insight into the anchoring of melittin in the DMPC bilayer. Residues at the $\mathrm{N}$ - and $\mathrm{C}$-termini are observed to form hydrogen bonds with the lipid head-groups of the surrounding lipids. In particular the positively charged C-terminus [i.e. side-chains of $\operatorname{Arg}(22)$ and $G \ln (25)$ ] provides an "anchor" to the bilayer via formation of multiple hydrogen bonds that persist throughout the simulation. The observation of hydrogen bonds between the side-chain of Lys(7) and the lipid head groups of DMPC deserves a special mention. The side-chain of Lys(7), located in the hydrophobic part of the bilayer, reaches along the transmembrane helix allowing the charged moiety of the side-chain to interact with lipid head-groups, while the aliphatic part of the lysine sidechain favourably interacts with the lipid tails. The extended nature of Lys(7) in DMPC is evident from Fig. 5, particularly in Panel C. This effect has also been observed in glycosylation mapping experiments to investigate the effects of single charged residues in transmembrane helices and was referred to as the "snorkel-effect" [78, 79]. It was found that charged residues with very long side-chains can snorkel down to interact with the lipid head-group region of the membrane.

\section{Conclusion}

We performed relatively long molecular dynamics simulations of the membrane active, $\alpha$-helical peptide melittin in three different environments, in aqueous solution (30 ns), in methanol (60 ns) and as a transmembrane helix embedded in a DMPC bilayer $(10+17 \mathrm{~ns})$. While the peptide is observed in water to collapse into a partly random, partly helical structure within the first $5 \mathrm{~ns}$, the peptide appears to be stable in methanol for $10 \mathrm{~ns}$ and is then observed to partly unfold from the $\mathrm{N}$-terminus to the proline residue at position 14. The remaining 13-residue long $\alpha$-helix is stable over the entire $60 \mathrm{~ns}$ of simulation. No refolding of the Nterminal part of the peptide is observed, it remains in a rather extended conformation throughout the simulation, which is somewhat contradictory to findings from NMR studies of melittin in methanol solution [19, 24]. In contrast to the simulations in solution, the $\alpha$-helical structure of melittin appears to be very stable when inserted in a DMPC-bilayer in a transmembrane orientation. Thus, the stability of melittin seems to increase when going from polar to apolar media as also observed by Liu and Hsu [43]. The investigation of peptide-lipid hydrogen bonds shows that the C-terminus of melittin forms multiple hydrogen bonds with the surrounding lipids, thus providing an anchor to the bilayer. 
Furthermore, interactions between the lysine side-chain of residue 7 and the lipid head group region are observed confirming the "snorkel" model of lysine residues in transmembrane helices introduced by Segrest et al. [78].

Acknowledgments Financial support was obtained through the National Center of Competence in Research (NCCR) Structural Biology of the Swiss National Science Foundation, which is gratefully acknowledged. A.G. thanks Dr. Bojan Zagrovic and Dr. Chris Oostenbrink for fruitful discussions.

\section{References}

1. Zasloff M (1992) Antibiotic peptides as mediators of innate immunity. Curr Opin Immunol 4:3-7

2. Bernheimer AW, Rudy B (1986) Interactions between membranes and cytolytic peptides. Biochim Biophys Acta 864:123141

3. White SH, Wimley WC, Selsted ME (1995) Structure, function, and membrane integration of defensins. Curr Opin Struct Biol 5:521-527

4. Bechinger B (1997) Structure and functions of channel-forming peptides: magainins, cecropins, melittin and alamethicin. J Membrane Biol 156:197-211

5. Tossi A, Sandri L, Giangaspero A (2000) Amphipathic, $\alpha$ helical antimicrobial peptides. Biopolymers 55:4-30

6. Pouny Y, Rapaport D, Mor A, Nicolas P, Shai Y (1992) Interaction of antimicrobial dermaseptin and its fluorescently labeled analogues. Biochemistry 31:12416-12423

7. Oren Z, Shai Y (1998) Mode of action of linear amphipathic $\alpha$ helical antimicrobial peptides. Biopolymers 47:451-463

8. Christensen B, Fink J, Merrifield RB, Mauzerall D (1988) Channel-forming peptides of cecropins and related model compounds incorporated into planar lipid-membranes. Proc Natl Acad Sci USA 85:5072-5076

9. Ludtke S, He K, Huang HW (1995) Membrane thinning caused by magainin 2. Biochemistry 34:16764-16769

10. Matsuzaki K, Murase O, Fujii N, Miyajima K (1995) Translocation of a channel-forming antimicrobial peptide, magainin 2 , across lipid bilayers by forming a pore. Biochemistry 34:6521-6526

11. Biggin PC, Sansom MSP (1999) Interactions of $\alpha$-helices with lipid bilayers: a review of simulation studies. Biophys Chem 76:161-183

12. Ladokhin AS, White SH (2001) "detergent-like" permeabilization of anionic lipid vesicles by melittin. Biochim Biophys Acta 1514:253-260

13. Habermann E (1972) Bee and wasp venoms. Science 177:314322

14. Yunes R, Goldhammer AR, Garner WK, Cordes EH (1977) Phospholipases-melittin facilitation of bee venom phospholipase A2 catalyzed hydrolysis of unsonicated lecithin liposomes. Arch Biochem Biophys 183:105-112

15. Shier WT (1979) Activation of high levels of endogenous phospholipase A2 in cultured cells. Proc Natl Acad Sci USA 76:195-199

16. Morgan CG, Williamson H, Fuller S, Hudson B (1983) Melittin induces fusion of unilamellar phospholipid-vesicles. Biochim Biophys Acta 732:668-674

17. Habermann E, Jentsch J (1967) Sequenzanalyse des Melittin aus den tryptischen und peptischen Spaltstücken. H- S Z Physiol Chem 348:37-50

18. Terwilliger TC, Eisenberg D (1982) The structure of melittin. I. Structure determination and partial refinement. J Biol Chem 257:6010-6015

19. Bazzo R, Tappin MJ, Pastore A, Harvey TS, Carver JA, Campbell ID (1988) The structure of melittin - a ${ }^{1} \mathrm{H}-\mathrm{NMR}$ study in methanol. Eur J Biochem 173:139-146
20. Inagaki F, Shimada I, Kawaguvhi K, Hirano M, Terasawa I, Ikura T, Go N (1989) Structure of melittin bound to perdeuterated dodecylphosphocholine micelles as studied by twodimensional NMR and distance geometry calculations. Biochemistry 28:5985-5991

21. Ikura T, Go N, Inagaki F (1991) Refined structure of melittin bound to perdeuterated dodecylphosphocholine micelles as studied by 2D-NMR and distance geometry calculation. Proteins: Struct Funct Genet 9:81-89

22. Brown LR, Wüthrich K (1981) Melittin bound to dodecylphosphocholine micelles $-{ }^{1} \mathrm{H}-\mathrm{NMR}$ assignments and global conformational features. Biochim Biophys Acta 647:95-111

23. Brown LR, Braun W, Kumar A, Wüthrich K (1981) High resolution nuclear magnetic resonance studies of the conformation and orientation of melittin bound to a lipid-water interface. Biophys J 37:319-328

24. Dempsey CE (1988) pH-Dependence of hydrogen-exchange from backbone peptide amides of melittin in methanol. Biochemistry 27:6893-6901

25. Terwilliger TC, Weissman L, Eisenberg D (1981) The structure of melittin in the form I crystals and its implication for melittin's lytic and surface activities. Biophys J 37:353-361

26. Lauterwein J, Brown LR, Wüthrich K (1980) High-resolution ${ }^{1} \mathrm{H}-\mathrm{NMR}$ studies of monomeric melittin in aqueous solution. Biochim Biophys Acta 622:219-230

27. Brown LR, Lauterwein J, Wüthrich K (1980) High-resolution ${ }^{1} \mathrm{H}-\mathrm{NMR}$ studies of self-aggregation of melittin in aqueous solution. Biochim Biophys Acta 622:231-244

28. Vogel H, Jähnig F (1986) The structure of melittin in membranes. Biophys J 50:573-582

29. Vogel H (1987) Comparison of the conformation and orientation of alamethicin and melittin in lipid membranes. Biochemistry 26:4562-4572

30. Stanislawski B, Rüterjans H (1987) ${ }^{13}$ C-NMR Investigation of the insertion of the bee venom melittin into lecithin vesicles. Eur Biophys J 15:1-12

31. Altenbach C, Froncisz W, Hyde JS, Hubbell WL (1989) Conformation of spin-labeled melittin at the membrane surfaces investigated by pulse saturation recovery and continuous wave power saturation electron paramagnetic resonance. Biophys $\mathrm{J}$ 56:1183-1191

32. Kuchinka E, Seelig J (1989) Interaction of melittin with phosphatidylcholine membranes. Binding isotherm and lipid headgroup conformation. Biochemistry 28:4216-4221

33. Beschiaschvili G, Seelig J (1990) Melittin binding to mixed phosphatidylglycerol/phosphatidylcholine membranes. Biochemistry 29:52-58

34. Frey S, Tamm LK (1991) Orientation of melittin in phospholipid bilayers - a polarized attenuated total reflection infrared study. Biophys J 60:922-930

35. Werkmeister JA, Kirkpatrick A, McKenzie JA, Rivett DE (1993) The effect of sequence variations and structure on the cytolytic activity of melittin peptides. Biochim Biophys Acta 1157:50-54

36. Rex S (1996) Pore formation induced by the peptide melittin in different lipid vesicle membranes. Biophys Chem 58:75-85

37. Rex S (2000) A Pro $\rightarrow$ Ala substitution in melittin affects self association, membrane binding and pore-formation kinetics due to changes in structural and electrostatics properties. Biophys Chem 85:209-228

38. Hristova K, Dempsey CE, White SH (2001) Structure, location, and lipid perturbations of melittin at the membrane interface. Biophys J 80:801-811

39. Lee M-T, Fang-Yu C, Huang H-W (2004) Energetics of pore formation induced by membrane active peptides. Biochemistry 43:3590-3599

40. Dempsey CE (1990) The actions of melittin in membranes Biochim Biophys Acta 1031:143-161

41. La Rocca P, Biggin PC, Tieleman DP, Sansom MSP (1999) Simulation studies of the interaction of antimicrobial peptides and lipid bilayers. Biochim Biophys Acta 1462:185-200 
42. Sessions RB, Gibbs N, Dempsey CE (1998) Hydrogen bonding in helical polypeptides from molecular dynamics simulations and amide hydrogen exchange analysis: alamethicin and melittin in methanol. Biophys $\mathbf{J}$ 74:138-152

43. Liu H-L, Hsu C-M (2004) The effects of solvent and temperature on the structural integrity of monomeric melittin by molecular dynamics simulations. Chem Phys Lett 375: $119-125$

44. Roccatano D, Colombo G, Fioroni M, Mark AE (2002) Mechanism by which 2,2,2-trifluorethanol/water mixtures stabilize secondary-structure formation in peptides: a molecular dynamics study. Proc Natl Acad Sci 99:12179-12184

45. Gerig JT (2004) Structure and solvation of melittin in 1,1,1,3,3,3-hexafluoro-2-propanol. Biophys J 86:3166-3175

46. Bernèche S, Nina M, Roux B (1998) Molecular dynamics simulation of melittin in dimyristoylphosphatidylcholine bilayer membrane. Biophys J 75:1603-1618

47. Bachar M, Becker OM (1999) Melittin at a membrane/water interface: Effects on water orientation and water penetration. J Chem Phys 111:8672-8685

48. Lin J-H, Baumgärtner A (2000) Molecular dynamics simulations of hydrophobic and amphipathic proteins interacting with a lipid bilayer membrane. Comp Theor Pol Sci 10:97-102

49. Lin J-H, Baumgärtner A (2000) Adsorption of melittin to a lipid bilayer: A molecular dynamics study. J Mol Liq 84:8998

50. Bachar M, Becker OM (2000) Protein-induced membrane disorder: a molecular dynamics study of melittin in a dipalmitoylphosphatidylcholine bilayer. Biophys J 78:1359-1375

51. Lin J-H, Baumgärtner A (2000) Stability of a melittin pore in a lipid bilayer: a molecular dynamics study. Biophys J 78:17141724

52. van Gunsteren WF, Billeter SR, Eising AA, Hünenberger PH, Krüger P, Mark AE, Scott WRP, Tironi IG (1996) Biomolecular Simulation: The GROMOS96 Manual and User Guide. vdf Hochschulverlag, ETH Zürich, Switzerland

53. Scott WRP, Hünenberger PH, Tironi IG, Mark AE, Billeter SR, Fennen J, Torda AE, Huber T, Krüger P, van Gunsteren WF (1999) The GROMOS biomolecular simulation program package. J Phys Chem 103:3596-3607

54. Schuler LD, Daura X, van Gunsteren WF (2001) An improved GROMOS96 force field for aliphatic hydrocarbons in the condensed phase. J Comput Chem 22:1205-1218

55. Chandrasekhar I, Kastenholz M, Lins RD, Oostenbrink C, Schuler LD, Tieleman DP, van Gunsteren WF (2003) A consistent potential energy parameter set for lipids: dipalmitoylphosphatidylcholine as a benchmark of the GROMOS 45A3 force field. Eur Biophys J 32:67-77

56. Chiu SW, Clark M, Subramaniam S, Scott HL, Jakobsson E (1995) Incorporation of surface tension into molecular dynamics simulation of an interface: A fluid phase lipid bilayer membrane. Biophys J 69:1230-1245

57. Terwilliger TC, Eisenberg D (1982) The structure of melittin. II. Interpretation of the structure. J Biol Chem 257:60166022

58. Berendsen HJC, Postma JPM, van Gunsteren WF, Hermans J (1981) Interaction models for water in relation to protein hydration. In: Pullman B (ed) Intermolecular forces. Reidel, Dordrecht, pp 331

59. Nagle JF, Tristram-Nagle S (2000) Structure of lipid bilayers. Biochim Biophys Acta 94(3):435
60. Yau WM, Wimley WC, Gawrisch K, White SH (1998) The preference of tryptophan for membrane interfaces. Biochemistry 37:14713-14718

61. Vogel H, Jähnig F, Hoffmann V, Stümpel J (1983) The orientation of melittin in lipid membranes. Biochim Biophys Acta 733:201-209

62. Berendsen HJC, Postma JPM, van Gunsteren WF, DiNola A, Haak JR (1984) Molecular dynamics with coupling to an external bath. J Chem Phys 81:3684-3690

63. Ryckaert J-P, Ciccotti G, Berendsen HJC (1977) Numerical integration of the cartesian equations of motion of a system with constraints: molecular dynamics of $n$-alkanes. J Comput Phys 23:327-341

64. Christopher JA, Swanson R, Baldwin TO (1996) Algorithms for finding the axis of a helix: fast rotational and parametric least-squares methods. Computers Chem 20:339-345

65. Kabsch W, Sander C (1983) Dictionary of protein secondary structure: pattern recognition of hydrogen bonded and geometrical features. Biopolymers 22:2577-2637

66. Karplus M (1959) Contact electron-spin coupling of nuclear magnetic moments. J Chem Phys 30:11-15

67. Pardi A, Billeter M, Wüthrich K (1984) Calibration of the angular dependence of the amide proton- $\mathrm{C}^{\alpha}$ proton coupling constants, ${ }^{3} \mathrm{~J}_{\mathrm{HN}} \alpha$, in a globular protein. J Mol Biol 180:741-751

68. Feig M, MacKerrell AD Jr, Brooks III CL (2004) Force field influence on the observation of $\pi$-helical protein structures in molecular dynamics simulations. J Phys Chem 107:2831-2836

69. Armen R, Alonso DOV, Daggett V (2003) The role of $\alpha-, 310^{-}$, and $\pi$-helix in helix $\rightarrow$ coil transitions. Protein Sci 12:11451157

70. Peter C, Rueping M, Wörner HJ, Jaun B, Seebach D, van Gunsteren WF (2003) Molecular dynamics of small peptides: can one derive conformational spectra from ROESY spectra Chem Eur J 9:5838-5849

71. Iwadate M, Asakura T, Dubovskii PV, Yamada H, Akasaka K, Williamson MP (2001) Pressure-dependent changes in the structure of the melittin $\alpha$-helix determined by NMR. J Biomol NMR 19:115-124

72. Chou PY, Fasman GD (1978) Prediction of the secondary structure of proteins from their amino acid sequence. Advan Enzymol 47:45-148

73. Barlow DJ, Thornton JM (1988) Helix geometry in proteins. J Mol Biol 201:601-619

74. von Heijne G (1991) Proline kinks in transmembrane $\alpha$-helices. J Mol Biol, 218:499-503

75. Cordes FS, Bright JN, Sansom MSP (2002) Proline-induced distortions of transmembrane helices. J Mol Biol, 323:951-960

76. Brandl CJ, Deber CM (1989) Hypothesis about the function of membrane-buried proline residues in transport proteins. Proc Natl Acad Sci 83:917-921

77. Woolfson DN, Mortishire-Smith RJ, Williams DH (1991) Conserved positioning of proline residues in membrane-spanning helices of ion-channel proteins. Biochem Biophys Res Commun 175:733-737

78. Segrest JP, De Loof H, Dohlmann JG, Brouilette CG, Anantharamaiah GM (1990) Amphipathic helix motif: classes and properties. Proteins: Struct Funct Genet 8:103-117

79. Monneé M, Nilsson I, Johansson M, Elmhed N, von Heijne G (1998) Positively and negatively charged residues have different effects on the position in the membrane of a model transmembrane helix. J Mol Biol 284:1177-1183 